\title{
Métodos de Evaluación del Rendimiento Masticatorio. Una Revisión
}

\author{
A Review of Different Masticatory Performance Tests \\ Schott Börger $S^{1}$, Ocaranza Tapia $D^{2}$, Peric Cáceres $K^{2}$, Yévenes López $I^{3}$, Romo Ormazábal $F^{1,2}$, \\ Schulz Rosales $\mathrm{R}^{1}$, Torres-Quintana MA $\mathrm{M}^{1,4}$
}

\begin{abstract}
RESUMEN
Desde comienzos del siglo pasado han sido utilizados una amplia variedad de métodos para evaluar el rendimiento masticatorio. Existen tests que usan alimentos naturales como material de prueba, otros utilizan alimentos artificiales; además se han propuesto distintos sistemas de procesamiento del material triturado, como el tamizado fraccional, espectrofotométrico, de aire comprimido, observación de área, concentración de azúcar, fotométrico y escaneo óptico. En este trabajo se describen y discuten algunos métodos de trascendencia en la historia de la evaluación del rendimiento masticatorio.
\end{abstract}

Rev. Clin. Periodoncia Implantol. Rehábil. Oral Vol. 3(1); 51-55, 2010.

Palabras clave: Tests masticatorios, función masticatoria, rendimiento masticatorio.

\section{ABSTRACT}

Masticatory performance has been assessed with varying methods throughout last century. Some of them use natural food as a test material and some others use artificial components instead of food. There have also been proposed various systems of processing the crushed material, such as fractional sieving, spectrophotometric analysis, compressed air, area observation, sugar concentration, photometric and optical scanning. This paper describes and discusses some relevant methods in the history of the evaluation of masticatory performance.

Rev. Clin. Periodoncia Implantol. Rehábil. Oral Vol. 3(1); 51-55, 2010.

Key words: Masticatory tests, masticatory function, masticatory performance.

\section{INTRODUCCIÓN}

La masticación es el primer paso de la digestión donde la comida se prepara para ser deglutida y luego procesada en el sistema digestivo ${ }^{(1,2)}$. Corresponde a un conjunto de fenómenos estomatognáticos destinados a fragmentar los alimentos en partículas pequeñas adecuadas para ser deglutidas ${ }^{(3)}$. Uno de los principales motivos de consulta odontológica es para mejorar la masticación y los tratamientos dentales deben devolver esa función y velar porque ésta se desarrolle en la forma más óptima posible, pero ¿cómo puede el odontólogo evaluar "la función masticatoria" en forma objetiva?

La Función masticatoria es un concepto unificador usado para referirse en forma global a términos como habilidad, eficiencia y/o rendimiento masticatorio y corresponde a la respuesta subjetiva de los pacientes acerca de su masticación y su capacidad objetiva para masticar $^{(4)}$. Sin embargo, los conceptos de eficiencia, rendimiento y habilidad masticatoria tienen definiciones diferentes que pueden ayudar al clínico a evaluar la función masticatoria. Eficiencia masticatoria corresponde al número de golpes masticatorios requeridos para lograr un nivel tipo de pulverización de un determinado alimento, por ejemplo el número de golpes necesarios para preparar el alimento a ser deglutido. Rendimiento masticatorio corresponde al grado de trituración al que puede ser sometido un alimento con un número dado de golpes masticatorios ${ }^{(5,6)}$. La habilidad masticatoria se define como la propia valoración de un individuo respecto a su masticación ${ }^{(4)}$.

De esta forma la habilidad masticatoria de un individuo puede ser evaluada por medio de cuestionarios o entrevistas personales como lo hacen G. Agerberg y G. E. Carlsson, 1981; T. Osterberg y B. Steen, 1982. Lamentablemente se trata de una valoración subjetiva donde no existe un enfoque universalmente aceptado por los investigadores y clínicos $^{(4,7)}$. La eficiencia y el rendimiento masticatorio, en cambio, pueden ser evaluados por medio de tests de masticación que proporcionan una valoración objetiva de la función masticatoria ${ }^{(4,8,9,10)}$ esta metodología puede usarse en forma efectiva siempre y cuando el test haya sido estandarizado(4,8). Es importante señalar que si bien algunos autores han encontrado una correlación significativa entre los tests masticatorios subjetivos y los objetivos, hay otros que han refutado esta postura, hallando una muy débil e incluso inexistente correlación entre ambos ${ }^{(4,8,10,11)}$

El propósito de este trabajo es describir y discutir algunos métodos de trascendencia en la historia de la evaluación del rendimiento masticatorio; entregando al lector información respecto a los métodos para su medición y su utilidad clínica. Aunque la mayoría de estos métodos son difíciles y engorrosos de realizar en la clínica cotidiana, algunos pueden resultar más simples y aplicables por el dentista para obtener valores de rendimiento masticatorio en sus pacientes. Se mencionan trabajos que revolucionaron los métodos de valoración del rendimiento masticatorio, es decir, que introdujeron cambios importantes e innovaciones en su desarrollo. Se trata de una revisión literaria, en la que se seleccionaron trabajos sobre rendimiento masticatorio desde Pubmed, incluyendo aquellos artículos que describieran detalladamente el método indagado.

\section{TESTS MASTICATORIOS}

Se han utilizado distintos tests masticatorios a lo largo de la historia. Entre los más utilizados, el tamizado fraccional como técnica para separar la comida después de ser masticada por un cierto período de tiempo, se ha usado desde 1924 y aún se considera un método viable ${ }^{(4)}$ Consiste en que el individuo mastica una porción medida de alimento de prueba con un número determinado de golpes masticatorios

1. Clínica Odontológica del Adulto, Facultad de Odontología Universidad de Chile. Chile.

2. Departamento de Prótesis, Facultad de Odontología Universidad de Chile. Chile.

3. Departamento de Ciencias Químicas y Físicas, Facultad de Odontología Universidad de Chile. Chile.

4. Departamento de Patología, Facultad de Odontología Universidad de Chile. Chile.

Financiado por proyecto PRI-ODO/07/001. 
o por un cierto período de tiempo. Luego se recupera el alimento test y se cuela a través de un tamiz. Se determina la masa o volumen de alimento remanente en el colador y el que pasa a través de él. La tasa de rendimiento masticatorio se define como la masa o volumen de alimento que pasa por el cedazo dividido por la masa o volumen total de comida recuperada, expresado como un porcentaje ${ }^{(12)}$.

Uno de los primeros intentos por crear un método que permita medir la función masticatoria es el de S. E. Gelman (1933). Determina el rendimiento masticatorio solicitándole a un sujeto a masticar una porción de 5 gramos de avellanas por 50 segundos sin instruirle el lado de la boca a usar. El alimento pulverizado es expectorado en un recipiente y tamizado a través de una gasa. Las partículas remanentes sobre la gasa se secan sobre un baño de agua por 40 minutos. Se filtran las partículas agitando la masa seca sobre un tamiz con una malla de $2.4 \mathrm{~mm}$ de apertura. Se masan las partículas que permanecen sobre el colador y se calcula el porcentaje de ellas en relación a la masa total del alimento ingerido. El coeficiente de eficiencia masticatoria se determina al restarle el valor obtenido a $100^{(13)}$

P. C. Carman (1938) desarrolló un método en el cual el aumento del área superficial es calculado por la disminución en la presión de aire comprimido al pasar a través del material triturado ${ }^{(14)}$

En 1942 B. Dahlberg plantea un nuevo método. Ensaya distintos materiales de prueba incluyendo clara de huevo hervida, zanahoria, caucho y gelatina decidiendo finalmente utilizar gelatina ${ }^{(15)}$. Le pide al paciente que mastique un pedazo de gelatina endurecida en formalina de $10.6 \mathrm{~mm}^{3}$ con 40 golpes masticatorios sin indicarle el lado de la boca a usar. La gelatina masticada es transferida a un aparato donde se cuela a través de 10 tamices con mallas con aperturas de $1 \mathrm{a}$ $10 \mathrm{~mm}$. Terminado este proceso se cuenta el número de partículas de cada cedazo. Se usa una fórmula especial para determinar el volumen total de las porciones, su área superficial y finalmente el coeficiente de masticación, calculado como superficie por unidad de volumen (milímetros cuadrados y cúbicos)(13).

Este autor listó un número de requisitos de un material de prueba ideal:

I. Debe asemejarse a un alimento natural, es decir, que no sea tan fácil de masticar como para poder ser triturado por los rebordes alveolares, pero tampoco tan difícil como para que las personas con una pobre dentición no puedan participar del test ${ }^{(15)}$.

II. No se debe abultar o disolver en agua o saliva y se debiera pulverizar de tal manera que el grado de pulverización se pueda establecer claramente ${ }^{(15)}$

III. No se debe fragmentar a través de líneas de clivaje predeterminadas o ser duro o pegajoso ${ }^{(15)}$

IV. Debe ser posible de estandarizar, ser no perecible y de buen sabor o insípido ${ }^{(15)}$

Uno de los tests que ha soportado la prueba del tiempo, siendo utilizado desde hace muchos años en una amplia escala en los trabajos de muchos investigadores, es el test R. S. Manly y L. C. Braley (1950). Éste utiliza maní salado como alimento de prueba y el resultado se obtiene a través de una filtración fraccional con un solo tamiz ${ }^{(13)} .15$ gramos de maní son divididos en 5 porciones de 3 gramos cada una. Cada porción es sometida a 20 golpes masticatorios y después introducida en un solo contenedor, agitada para romper las aglomeraciones y lavada con $500 \mathrm{cc}$ de agua por un tamiz de malla con aperturas de $1.7 \mathrm{~mm}$. Las partículas que permanecen en la malla y las partículas que pasan por el tamiz son filtradas en hojas separadas de papel filtro. Cada fracción obtenida es secada en un horno a $100^{\circ} \mathrm{C}$ por 3 horas, transferida a un desecador por 2 horas y luego masada. El rendimiento masticatorio se calcula como el porcentaje del alimento masticado masado que pasa por el tamiz en relación con el total de alimento masado recuperado de la boca ${ }^{(5,13)}$. Se les permite a los individuos triturar las porciones del alimento de prueba a su propia y habitual manera, sin ninguna instrucción como el lado de la boca a utilizar para masticar ${ }^{(13)}$.

Un par de meses posterior a esta publicación A. Yurkstas y R. S. Manly (1950) proponen modificaciones al método planteado originalmente por uno de los autores. Ellos plantean la posibilidad de someter las partículas que permanecen en la malla y las que pasan por el tamiz a un proceso de centrifugación con el fin de separar el contenido líquido del material de prueba y de esta manera determinar el rendimiento masticatorio como el volumen de partículas que pasan por el tamiz dividido por el volumen total de alimento colectado de la boca. Logran introducir un método alternativo al sistema de masado. Ellos simplifican el método anterior sometiendo 3 porciones de 3 gramos cada una (en total 9 porciones de maní) a la masticación. Además prueban 35 distintos alimentos naturales como material de prueba y proponen al jamón, a las ciruelas secas y zanahorias como otros materiales válidos alternativos al maní(16).

En 1963 S. Loos crea un método utilizando almendras como material de prueba las que se analizan luego de ser trituradas al ser esparcidas en papel cuadriculado(17). Este procedimiento es posteriormente modificado por E. Helkimo, G. E. Carlsson y M. Helkimo (1978). Estos últimos procesaron las almendras usando un tamiz y clasificaron la eficiencia en una escala del 1 al 5 , siendo 1 una muy buena y 5 una muy pobre habilidad para reducir las partículas del alimento de prueba $^{(18)}$.

K. K. Kapur, S. D. Soman y A. Yurkstas en 1964 prueban la utilidad de la zanahoria como alimento de prueba. Utilizan un sistema en el que el sujeto mastica tres porciones previamente medidas de alimento de prueba un número de golpes masticatorios específicos ( 20 golpes para maní y 40 para zanahorias). El alimento se recupera y se tamiza a través de un cedazo de malla estandarizada $(1.7 \mathrm{~mm}$ para maní y $4 \mathrm{~mm}$ para zanahoria). Se determina el volumen de alimento remanente en el tamiz y el que pasa a través de éste. La tasa de rendimiento masticatorio se define como el volumen de alimento que pasa por el tamiz, dividido por el volumen total de partículas recuperadas, expresado como porcentaje ${ }^{(19)}$.

A. F. Käyser y J. S. van der Hoeven el año 1977 inician los métodos espectrofotométricos creando un método basado en la tinción liberada naturalmente de zanahorias crudas sometidas a la masticación. Esta tinción era medida espectrofotométricamente a una longitud de onda determinada. El sujeto era instruido a masticar un trozo de zanahoria de 3 gramos con un número determinado de golpes. Todo el material triturado durante el proceso más la saliva acumulada es expectorado en un cilindro graduado. Luego se le agrega agua y se agita con magneto. Posteriormente todo el contenido era colocado sobre papel filtro y el filtrado analizado en espectrofotómetro. La absorbancia por gramo de zanahoria era calculada y el resultado era expresado como porcentaje de la máxima cantidad de tinte liberado desde el vegetal(17).

J. Edlund y C. J. Lamm (1980) proponen el uso de un alimento artificial una silicona de condensación (de impresión) como material de prueba. Ellos escogieron un producto llamado Optosil $\Omega_{\text {que luego }}$ cambió a CutterSil ${ }^{\circledR}$. CutterSil $\circledast$ tiene muy poco sabor y olor, no se afecta con el agua, y se puede almacenar por 7 días sin perder estabilidad dimensional. Adicionalmente no tiene líneas de clivaje predeterminadas, se puede examinar fácilmente después de la masticación y se puede conformar fácilmente con tamaños y masas estándares. Eso sí se le debe dar gran atención a la mezcla del material, pues es un paso trascendental para obtener propiedades estandarizadas, especialmente de dureza ${ }^{(20)}$ El método original de J. Edlund y C. J. Lamm (1980) ha sido modificado por diversos autores: S. M. Omar, J. D. McEwen y S. A. Ogston (1987); K. C. Julien et al. (1996) y P. H. Buschang et al. $(1997)^{(20)}$. Instruyen a los sujetos a masticar la porción de prueba previamente masada, de $5 \mathrm{~mm}$ de grosor y de $20 \mathrm{~mm}$ de diámetro lo más completamente posible con 20 golpes masticatorios. Se les permite masticar uni o bilateralmente. E bolo es expectorado en una copa plástica, el paciente se enjuaga con agua $(100 \mathrm{~mL})$ y es examinado por el operador para cerciorar la ausencia de partículas remanentes. Este proceso se repite 5 veces. Se retira con papel filtro el agua y la saliva de las partículas y luego se introducen en un horno para ser secadas. Luego se colocan las partículas en un sistema de tamices (con aperturas de $5.6 \mathrm{~mm}, 4 \mathrm{~mm}, 2.8 \mathrm{~mm}$ y $2 \mathrm{~mm}$ ) con vibrador por 120 segundos. Finalmente mediante un índice de eficiencia masticatoria se calcula el rendimiento masticatorio caracterizado por la distribución del material de prueba en las distintas fracciones ${ }^{(15,21)}$

En 1982 M. R. Heath desarrolla un método usando goma de mascar. Utiliza un rectángulo de 1 gramo de una goma de consumo popular en su país (Wrigley's Doublemint ${ }^{\circledR}$ o Wrigley's Freedent Peppermint $($ ) que es sometida a 20 golpes masticatorios por parte del paciente. La goma de mascar es recuperada, disecada y masada nuevamente. Luego se calcula la eficacia masticatoria con el porcentaje de azúcar extraído. Este test probó ser de fácil aplicación y de aceptación popular (22). Además este test evita las molestias ocasionadas por las partículas de algunos alimentos de prueba al introducirse bajo la base protésica, permite recuperar el material de prueba en su totalidad y discrimina entre distintos tipos de denticiones. Pero, si bien las gomas de mascar manufacturadas tienen la gran ventaja de ser estandarizadas, lamentablemente la composición es a veces reformulada. Además existe una estrecha correlación entre la tasa de secreción salival del paciente y los valores de eficacia obtenidos ${ }^{(23)}$

En 1982 H. S. Gunne et al. idean un nuevo método. Utilizan 3 cubos de gelatina endurecida en formalina de $2 \mathrm{~cm}^{3}$ que contienen un pigmento: fucsina. El primer cubo es masticado por el paciente por 10 segundos (de entrenamiento), el segundo cubo por 20 segundos y el tercer cubo hasta sentir que ya puede ser deglutido. El paciente puede masticar de la forma que desee. El supervisor cuenta el número de golpes masticatorios a los que son sometidos los cubos. A continuación se tamiza la sustancia masticada a través de una serie de 5 cedazos con mallas de distinta apertura $(7.85,5.80,3.80,3.10$ y 2.30$)$. Así se obtienen 5 fracciones por cada trozo de prueba, almacenados en recipientes de vidrio que se colocan en un horno a $110^{\circ} \mathrm{C}$ por 20 horas. Se determina 
entonces la masa seca del material de prueba y luego la masa se convierte en porcentaje de la masa original ${ }^{(24)}$.

Un año más tarde H. S. Gunne (1983) modifica su método publicado previamente. Los cubos de gelatina endurecida en formalina (con eritrosina en vez de fucsina) después de ser masticados se ponen en un tinte soluble en agua; el tinte difunde en las partículas y, por lo tanto, la concentración del tinte en la solución circundante disminuye. Así la variación en la concentración de la solución de tinción circundante va a ser proporcional al área de las partículas. Como el área es una medida de la trituración del material de prueba, el cambio en la concentración de tinte se puede usar para calcular la eficiencia masticatoria. Para esto se usó un espectrofotómetro(25)

En 1984 L. W. Olthoff et al. describen un método para medir el rendimiento masticatorio basado en el análisis detallado de la trituración de las partículas de alimento durante la masticación en función del número de golpes utilizados. Utilizan Optosil囚 como alimento de prueba y un método de tamizado estandarizado. La distribución por tamaño de las partículas alcanzado por los sujetos dentados se puede describir con una función matemática. A. van der Bilt et al. en 1987 publican un trabajo en el que describen este modelo matemático(26,27,28).

En 1992 A. P. Slagter et al. reducen el tamaño del cubo de Optosil@ y la cantidad de porciones ofrecidas utilizados por L. W. Olthoff et al. (1984) para poder comparar la trituración de portadores de prótesis totales con individuos con dentadura natural. Utilizan el método por tamizado en el procesamiento de las muestras ${ }^{(29)}$. En 1993 A. P. Slagter, F. Bosman y A. van der Bilt demuestran que Optocal $\AA$, un material de prueba basado en componentes similares a Optosil囚, es más fácil de triturar (posee una baja resistencia a la fractura) y, por lo tanto, más adecuado para medir el rendimiento masticatorio en pacientes portadores de prótesis totales ${ }^{(30)}$.

Como método alternativo al tamizado en 1989 A. Nakasima, $\mathrm{K}$. Higashi y $\mathrm{M}$. Ichinose diseñaron un método espectrofotométrico; establecen que el material de prueba debe tener propiedades físicas constantes, ser insoluble en agua y que el grado de fraccionamiento debe poder ser claramente establecido. Además todo el material masticado en boca se ha de permitir recuperar fácilmente. Posteriormente el análisis de laboratorio debe ser simple ${ }^{(14)}$. Los autores propusieron utilizar una cápsula de látex rellena de un granulado sintético que es masticada por el paciente con 15 golpes masticatorios (uno por segundo); así los gránulos se fracturan y se libera el pigmento rojo dentro de la cápsula en proporción a la energía usada. Luego se calcula el rendimiento masticatorio midiendo la concentración de colorante contenida en las cápsulas en una solución de agua mediante un espectrofotómetro ${ }^{(14)}$. Los gránulos especialmente diseñados para el estudio, tienen propiedades físicas estables y permanecen inmunes a la humedad dentro de las cápsulas de caucho, así se puede recuperar para ser medido el $100 \%$ de la masa original. La cápsula es insípida e inodora y se parece a goma de mascar, pudiendo ser masticada por personas con distintas condiciones dentales y orales. Lamentablemente la cápsula de caucho no representa todos los alimentos de varios tamaños, formas y rigidez, por lo tanto, sólo se puede evaluar un aspecto de la masticación ${ }^{(14)}$

En 1993 A. van der Bilt et al. proponen el escaneo óptico de las partículas de prueba (Optosil囚) con una cámara digital y un sistema de procesamiento de datos que analiza el diámetro de cada una de las partículas trituradas ${ }^{(31)}$. Ese mismo año $\mathrm{F}$. Mowlana y R. Heath utilizan un alimento de prueba natural como son las almendras envueltas en sacos de caucho para evitar la pérdida que ocurre tradicionalmente con el material de prueba y para aislar las partículas masticadas de la acción de la saliva para prevenir la cohesión; analizan la trituración de las partículas utilizando el escaneo óptico ${ }^{(32,33)}$

En comparación con el tamizado, el análisis de imagen de las partículas trituradas ofrece considerables ventajas como mayor simplicidad, velocidad, exactitud, reproducibilidad e higiene, tanto durante la aplicación del test como durante el procesamiento de las muestras. Es de fácil empleo para el paciente y para el examinador y le permite al examinado comprender fácilmente en forma visual los resultados del test. Este método es muy práctico para medir una gran cantidad de muestras ${ }^{(4,7,34)}$. Posee eso sí la desventaja de requerir separar las partículas del alimento unas de otras antes del escaneo y por ello consume bastante tiempo cuando existen muchas partículas; además el escaneo óptico sólo se puede aplicar en un pequeño número de partículas mayores a $1 \mathrm{~mm}$ de diámetro ${ }^{(32,35)}$.

En 1996 Y. Matsui et al. y luego en 1998 I. Hayakawa et al. investigan la utilidad para medir rendimiento masticatorio de una goma de mascar que varía de color. Los cambios de color que sufre la goma luego de ser masticada son medidos con el espacio de color $\mathrm{L}^{*} \mathrm{a}^{*} \mathrm{~b}^{*}$ definido por la Commission Internationale de l'Eclairage mediante el uso de un aparato que mide características ópticas. Desarrollan una escala de color y valores numéricos para evaluar fácilmente el cambio de color $^{(36,37)}$. El año $2003 \mathrm{H}$. Sato et al. basados en el trabajo de Hayakawa et al. (1998) usan unos cubos de cera de parafina de $12 \times 12 \times 12 \mathrm{~mm}$ de dimensión, con 6 capas coloreadas de rojo alternadas con otras 6 coloreadas de verde, que deben ser mantenidos en una incubadora a $37^{\circ} \mathrm{C}$ justo antes de ser usados. Luego se entregan al paciente para que los mastique un número determinado de veces según su masticación habitual. Una vez recuperadas, las muestras trituradas son analizadas mediante un análisis de imagen digital. Se toman imágenes de los cubos molidos con una cámara digital y con un analizador de imagen conectado a la máquina se observan parámetros colorimétricos y morfológicos, los que permiten determinar el rendimiento masticatorio ${ }^{(7)}$

En 1997 J. Huggare y B. Skindhöj realizando un trabajo similar al de H. S. Gunne (1983), proponen un método en el que incorporan un agente de enlace catiónico en el material de prueba (compuesto por cera y sulfato de bario) siendo este último masticado libremente con 10 golpes y luego lo introducen en un filtro de fibra de vidrio sobre un embudo. Luego de la filtración las partículas y el filtro son transferidas a un recipiente con solución de eritrosina con sustancias aniónicas. El colorante de la solución se adhiere a la superficie de las partículas de material triturado como un complejo insoluble. La absorción de color es proporcional al área superficial total del material masticado y al rendimiento masticatorio. La absorción de colorante se determina espectrofotométricamente como la diferencia en la absorbancia entre la solución de color estándar y la solución de color tratada con el material de $\operatorname{prueba}^{(35)}$.

G. Schneider y B. Senger en los años 2001 y 2002 mencionan en sus estudios que los materiales de prueba naturales no se deben dejar de lado para determinar el rendimiento masticatorio, fundamentados en que estos permiten poner al paciente en una situación del diario vivir Ellos analizan la utilidad de los granos de café y los proponen como una alternativa que merece ser considerada ${ }^{(38,39)}$.

En el año 2003 S. Okiyama, K. Ikebe y T. Nokubi determinan el rendimiento masticatorio con el aumento de gelatina disuelta desde la superficie de unas gelatinas gomosas usadas como material estandarizado de prueba. Los sujetos son instruidos a masticar la gelatina gomosa con 20 golpes masticatorios en su lado preferido de masticación (izquierdo o derecho) y a expectorar el bolo de partículas trituradas lo más cuidadosamente posible sobre una gasa. Las partículas colectadas se lavan con agua corriente por más de 30 segundos para remover la saliva. Se utiliza agua a $20^{\circ} \mathrm{C}$, lo suficientemente fría como para prevenir la disolución de la gelatina. Luego se remojan las partículas con $100 \mathrm{~mL}$ de agua destilada a $30-35^{\circ} \mathrm{C}$ y se agitan por 60 segundos. Posteriormente se toma una muestra del fluido sobrenadante de la solución, se diluye hasta 20 veces su volumen en agua destilada y se mide la concentración de gelatina disuelta con un espectrofotómetro. Primero se agregan $0.3 \mathrm{~mL}$ de un compuesto químico a cada muestra para aumentar la sensibilidad al colágeno por el método de Bradford de ensayo proteico de unión a tinción. Luego se agregan a la solución 0.6 $\mathrm{mL}$ de reactivo de tinción de prueba proteica. Se usa agua destilada $(2.4 \mathrm{~mL})$ de la misma manera como control. Luego de una incubación a temperatura ambiente por 30 minutos, la concentración de cada muestra de tinción es medida con un espectrofotómetro a $650 \mathrm{~nm}$ de longitud de onda ${ }^{(40)}$.

En el año $2006 \mathrm{~K}$. Ikebe et al. basados en el método de S. Okiyama, K lkebe y T. Nokubi (2003) determinan el rendimiento masticatorio con la concentración de glucosa disuelta obtenida desde las gelatinas gomosas usadas como material estandarizado de prueba. Los sujetos son instruidos a masticar la gelatina gomosa con 30 golpes masticatorios en su lado preferido de masticación (izquierdo, derecho o ambos) y a expectorar el bolo de partículas trituradas lo más cuidadosamente posible. Las partículas colectadas se lavan con agua corriente por más de 30 segundos para obtener un valor exacto. Luego se remojan con $15 \mathrm{~mL}$ de agua destilada y se agitan. Posteriormente se toma una muestra del fluido sobrenadante de la solución y se mide la concentración de glucosa disuelta de la gelatina gomosa fraccionada con un medidor de glucosa sanguíneo portable. El rendimiento masticatorio se determina al calcular el área superficial de las partículas desde la concentración de glucosa ${ }^{(41,42)}$.

En el año 2007 M. Schimmel et al. deciden simplificar la etapa del análisis de las gomas de mascar trituradas. Proponen sustituir los complejos y caros programas computacionales usados para analizar las muestras sometidas a la masticación por uno de fácil accesibilidad comercial como lo es Adobe Photoshop $®$. Sus resultados avalan la utilización de este producto para el análisis de muestras ${ }^{(9)}$.

\section{DISCUSIÓN}

La masticación es una de las funciones más importantes del sistema estomatognático. A comienzos del siglo XX los clínicos e investigadores notaron la necesidad de crear un método para evaluarla y 
que permita también realizar una evaluación objetiva de los tratamientos odontológicos. El interés se volcó en el estudio del rendimiento y la eficiencia masticatoria que entregan información objetiva de la función masticatoria. Para tal efecto se han desarrollado desde entonces una amplia gama de métodos, usando variados materiales o empleando algunas tecnologías similares.

Existen tests que utilizan alimentos naturales como material de prueba, otros, alimentos artificiales. Los alimentos naturales más utilizados han sido escogidos por tener poca variabilidad y, por lo tanto, ser buenos indicadores de diferencias entre las formas oclusales al masticar ${ }^{(8)}$. La ventaja de estos alimentos es que, al ser consumidos habitualmente, los sujetos que realizan el test están acostumbrados a masticarlos ${ }^{(43)}$, pero en cada ocasión, es difícil garantizar homogeneidad de las propiedades físicas de los alimentos. Se deben considerar ciertos factores como la época de cosecha, año e incluso el país de procedencia. Estos materiales se disuelven rápidamente en saliva o agua, de modo que una parte de ellos puede perderse al tragar y otra al momento de filtrarse luego de ser removidos de la boca. Debido al contenido de agua, el grado de pulverización también varía cuando las fracciones se secan para ser masadas $^{(14)}$. Estas desventajas han generado la necesidad de contar con nuevos materiales para los tests, creándose los "alimentos artificiales". Estos alimentos de prueba artificial son ventajosos, pues son inodoros e insípidos, además de tener propiedades físicas constantes ${ }^{(7,14)}$.

Los sistemas de procesamiento que permiten el análisis del material de prueba tras ser sometido a la masticación, también varían entre los distintos tests. Se incluyen el tamizado fraccional, espectrofotométrico, de aire comprimido, observación de área, concentración de azúcar, fotométrico y escaneo óptico. La mayoría de los investigadores han determinado el grado de fraccionamiento usando un sistema de tamices que incluyen filtrado, secado, masado y análisis; este método es complicado y consume mucho tiempo y, por lo tanto, no es adecuado para el uso clínico de rutina ${ }^{(7,14)}$. Además, otro de los errores de este sistema, es que la determinación del rendimiento masticatorio se basa en el porcentaje de masa seca del material masticado restante en el cedazo(14). Muchos trabajos que se han realizado determinan el rendimiento masticatorio usando el test de R. S. Manly y L. C. Braley $(1950)^{(44-55)}$ el cual usa un alimento natural como material de prueba y tamizado para su análisis, lo que hace difícil y engorroso para el paciente y el odontólogo la realización sistemática de este test.

El mundo odontológico, clínicos e investigadores tienen la necesidad de contar con un test de fácil aplicación clínica, que permita realizar una evaluación objetiva de los tratamientos odontológicos restauradores y ortodóncicos para demostrar al paciente las mejorías alcanzadas con los distintos tratamientos rehabilitadores y ayudar a discernir entre distintos planes de tratamiento. Debe medir la función masticatoria en la amplia gama de pacientes que acuden a la atención dental: niños, adultos o ancianos, hombres o mujeres, desdentados completos, dentados completos o dentados parciales, tomando en cuenta la disponibilidad de recursos financieros, humanos y materiales siempre limitados y cumpliendo los requisitos en lo que a viabilidad o factibilidad se refiere. No existe actualmente un test unificado, cada uno de los existentes tiene ventajas y desventajas, pero como hemos visto se han hecho muchos intentos por alcanzarlo y aún se continúa en su búsqueda, apoyados hoy en gran medida en la tecnología

El método propuesto en 1989 por A. Nakasima, K. Higashi y $\mathrm{M}$. Ichinose en que el material de prueba es un alimento artificial estandarizable con propiedades físicas constantes, parece un método reproducible e interesante, pues permanece libre de humedad dentro de cápsulas de caucho; por lo mismo además se puede recuperar toda la masa original para ser medida y permite un análisis del material triturado bastante ulterior a la aplicación del test. La cápsula es insípida e inodora y se parece a goma de mascar, pudiendo ser masticada por personas con distintas condiciones dentales y orales. Luego el análisis de laboratorio es simple, pudiendo establecerse claramente el grado de fraccionamiento del material de prueba con un espectrofotómetro.

\section{CONCLUSIONES}

- La masticación es una función que puede ser evaluada objetivamente midiendo el rendimiento y la eficiencia masticatoria.

- Se han desarrollado múltiples métodos usando variados materiales (alimentos naturales 0 artificiales) con diferentes sistemas de procesamiento, que han ido evolucionando con los avances en la tecnología.

- No existe actualmente un método unificado para medir el rendimiento masticatorio.

- Tal vez el método realizado por A. Nakasima, K. Higashi y M. Ichinose (1989) sea el que tiene la mayor aplicabilidad, pues permite el análisis del material en forma diferida, limpia y sin pérdida de material.

- No es posible comparar resultados de un test masticatorio con otro, pues no existen estudios que establezcan equivalencias entre los diferentes métodos.

- La obtención de un método simple para el odontólogo y no engorroso para el paciente puede permitir la realización sistemática de análisis del rendimiento masticatorio, lo que puede entregar valiosa información respecto a las evaluaciones de tratamientos odontológicos restauradores.

\section{REFERENCIAS BIBLIOGRÁFICAS}

1. van der Bilt $A$, Olthoff $L W$, Bosman $F$, Oosterhaven SP. Chewing performance before and after rehabilitation of post-canine teeth in man. J Dent Res. 1994 Nov;73(11):1677-83.

2. van der Bilt A, Olthoff LW, Bosman F, Oosterhaven SP. The effect of missing postcanine teeth on chewing performance in man. Arch Oral Biol. 1993 May;38(5):423-9.

3. Escudeiro Santos C, de Freitas O, Spadaro AC, Mestriner-Junior W. Development of a colorimetric system for evaluation of the masticatory efficiency. Braz Dent J. 2006;17(2):95-9.

4. Boretti G, Bickel M, Geering AH. A review of masticatory ability and efficiency. J Prosthet Dent. 1995 Oct; 74(4):400-403.

5. Manly RS, Braley LC. Masticatory performance and efficiency. J Dent Res. 1950 Aug;29(4):448-62

6. Manns, A., Díaz, G. Sistema Estomatognático. Facultad de Odontología - Universidad de Chile, 1995, 249 p. p.59-90, Capítulo II.

7. Sato H, Fueki K, Sueda S, Sato S, Shiozaki T, Kato M, et al. A new and simple method for evaluating masticatory function using newly developed artificial test food. J Oral Rehabil. 2003 Jan;30(1):68-73.

8. Demers M, Bourdages J, Brodeur JM, Benigeri M. Indicators of masticatory performance among elderly complete denture wearers. J Prosthet Dent. 1996 Feb;75(2):88-93.

9. Schimmel M, Christou P, Herrmann F, Müller F. A two-colour chewing gum test for masticatory efficiency: development of different assessment methods. J Oral Rehabil. 2007 Sep;34(9):671-8

10. Ikebe K, Morii K, Matsuda K, Nokubi T. Discrepancy between satisfaction with mastication, food acceptability, and masticatory performance in older adults. Int J Prosthodont. 2007 Mar-Apr;20(2):161-7.

11. Pereira LJ, Duarte Gaviao MB, van der BiltA. Influence of oral characteristics and food products on masticatory function. Acta Odontol Scand. 2006 Aug;64(4):193-201.
12. Tatematsu M, Mori T, Kawaguchi T, Takeuchi K, Hattori M, Morita I, et al. Masticatory performance in 80-year-old individuals. Gerodontology. 2004 Jun;21(2):112-9.

13. Krysinski Z, Ludwiczak T, Mucha J., Comparative investigations of selected methods of evaluating the masticatory ability. J Prosthet Dent. 1981 Nov;46(5):568-74.

14. Nakasima A, Higashi K, Ichinose M. A new, simple and accurate method for evaluating masticatory ability. J Oral Rehabil. 1989 Jul;16(4):373-80

15. Edlund J, Lamm CJ. Masticatory efficiency. J Oral Rehabil. 1980 Mar;7(2):123-30.

16. Yurkstas A, Manly RS. Value of different test foods in estimating masticatory ability. $J$ Appl Physiol. 1950 Jul;3:45-53.

17. Käyser AF, van der Hoeven JS. Colorimetric determination of the masticatory performance. J Oral Rehabil. 1977 Apr;4(2):145-8.

18. Helkimo E, Carlsson GE, Helkimo M. Chewing efficiency and state of dentition. A methodologic study. Acta Odontol Scand. 1978;36(1):33-41.

19. Kapur KK, Soman SD. Masticatory performance and efficiency in denture wearers. 1964. J Prosthet Dent. 2006 Jun;95(6):407-11.

20. Albert TE, Buschang PH, Throckmorton GS. Masticatory performance: a protocol for standardized production of an artificial test food. J Oral Rehabil. $2003 \mathrm{Jul} ; 30$ (7):720-2.

21. Omar SM, McEwen JD, Ogston SA. A test for occlusal function. The value of a masticatory efficiency test in the assessment of occlusal function. $\mathrm{Br} \mathrm{J}$ Orthod. 1987 Apr;14(2):85-90.

22. Heath MR. The effect of maximum biting force and bone loss upon masticatory function and dietary selection of the elderly. Int Dent J. 1982 Dec;32(4):345-56.

23. Anastassiadou V, Heath MR. The development of a simple objective test of mastication suitable for older people, using chewing gums. Gerodontology. 2001 Dec;18(2):79-86. 24. Gunne H.S., Bergman B., Enbom L., Högström J. Masticatory efficiency of complete denture patients. A clinical examination of potential changes at the transition from old to 
new dentures. Acta Odontol Scand. 1982;40(5):289-97.

25. Gunne H.S. Masticatory efficiency. A new method for determination of the breakdown of masticated test material. Acta Odontol Scand. 1983 Oct;41(5):271-6.

26. van der Bilt A, Olthoff LW, van der Glas HW, van der Weelen K, Bosman F. A mathematical description of the comminution of food during mastication in man. Arch Oral Biol. 1987;32(8):579-86.

27. Slagter AP, Olthoff LW, Bosman F, Steen WH. Masticatory ability, denture quality, and oral conditions in edentulous subjects. J Prosthet Dent. 1992 Aug;68(2):299-307.

28. Olthoff $L W$, van der Bilt A, Bosman F, Kleizen HH. Distribution of particle sizes in food comminuted by human mastication. Arch Oral Biol. 1984;29(11):899-903.

29. Slagter AP, Olthoff LW, Steen WH, Bosman F. Comminution of food by completedenture wearers. J Dent Res. 1992 Feb;71(2):380-6.

30. Slagter AP, Bosman F, van der Bilt A. Comminution of two artificial test foods by dentate and edentulous subjects. J Oral Rehabil. 1993 Mar;20(2):159-76.

31. van der Bilt A, van der Glas HW, Mowlana F, Heath MR. A comparison between sieving and optical scanning for the determination of particle size distributions obtained by mastication in man. Arch Oral Biol. 1993 Feb;38(2):159-62.

32. Al-Ali F, Heath MR, Wright PS. Simplified method of estimating masticatory performance. J Oral Rehabil. 1999 Aug;26(8):678-83.

33. Mowlana F, Heath R. Assessment of masticatory efficiency: new methods appropriate for clinical research in dental practice. Eur J Prosthodont Restor Dent. 1993 Mar; 1(3):121-5.

34. Mowlana F, Heath MR, van der Bilt A, van der Glas HW. Assessment of chewing efficiency: a comparison of particle size distribution determined using optical scanning and sieving of almonds. J Oral Rehabil. 1994 Sep;21(5):545-51.

35. Huggare J, Skindhöj B. A new method for assessing masticatory performance: a feasibility and reproducibility study. J Oral Rehabil. 1997 Jul;24(7):490-5.

36. Matsui Y, Ohno K, Michi K, Hata H, Yamagata K, Ohtsuka S. The evaluation of masticatory function with low adhesive colour-developing chewing gum. J Oral Rehabil. 1996 Apr;23(4):251-6.

37. Hayakawa I, Watanabe I, Hirano S, Nagao M, Seki T. A simple method for evaluating masticatory performance using a color-changeable chewing gum. Int J Prosthodont. 1998 Mar-Apr;11(2):173-6.

38. Schneider G, Senger B. Coffee beans as a natural test food for the evaluation of the masticatory efficiency. J Oral Rehabil. 2001 Apr;28(4):342-8.

39. Schneider G, Senger B. Clinical relevance of a simple fragmentation model to evaluate human masticatory performance. J Oral Rehabil. 2002 Aug;29(8):731-6.

40. Okiyama S, Ikebe K, Nokubi T. Association between masticatory performance and maximal occlusal force in young men. J Oral Rehabil. 2003 Mar;30(3):278-82.

41. Ikebe K, Matsuda K, Morii K, Furuya-Yoshinaka M, Nokubi T, Renner RP. Association of masticatory performance with age, posterior occlusal contacts, occlusal force, and salivary flow in older adults. Int J Prosthodont. 2006 Sep-Oct;19(5):475-81.

42. Ikebe K, Amemiya M, Morii K, Matsuda K, Furuya-Yoshinaka M, Yoshinaka M, et al. Association between oral stereognostic ability and masticatory performance in aged complete denture wearers. Int J Prosthodont. 2007 May-Jun;20(3):245-50.
43. Gambarelli FR, Serra MD, Pereira LJ, Duarte Gaviao MB. Influence of measurement technique, test food, teeth and muscle force interactions in masticatory performance. $\mathrm{J}$ Texture Stud. 2007 Feb;38(1):2-20.

44. Bolivar Figueroa J. Test de Manly y área masticatoria funcional en pacientes infantiles con problemas de oclusión. Trabajo de investigación Requisito para optar al título de Cirujano-Dentista. Universidad de Chile, Facultad de Odontología. Santiago, 1968.

45. Pozo M. Valoración clínica de eficiencia masticatoria en pacientes portadores de prótesis parcial removible. Trabajo de investigación Requisito para optar al título de Cirujano-Dentista. Universidad de Chile, Facultad de Odontología. Santiago, 1968.

46. Castro JC. Rendimiento masticatorio en la rehabilitación del paciente desdentado completo, según tipo de superficie masticatoria. Trabajo de investigación Requisito para optar al título de Cirujano-Dentista. Universidad de Chile, Facultad de Odontología. Santiago, 1988.

47. Hermosilla Gamero A. Análisis comparativo del rendimiento masticatorio en pacientes desdentados parciales de extremo libre inferior, rehabilitados en base a tres técnicas distintas de impresiones funcionales. Trabajo de investigación Requisito para optar al título de Cirujano-Dentista. Universidad de Chile, Facultad de Odontología. Santiago, 1993.

48. León Araya S. Estudio comparativo de rendimiento masticatorio entre el plano de oclusión superior y el plano de oclusión inferior. Trabajo de investigación Requisito para optar al título de Cirujano-Dentista. Universidad de Chile, Facultad de Odontología. Santiago, 1993.

49. Schulz Rosales R. Piezografía en la rehabilitación del desdentado completo: su influencia sobre la función masticatoria. Trabajo de investigación Requisito para optar al título de Cirujano-Dentista. Universidad de Chile, Facultad de Odontología. Santiago, 1993.

50. Fantela González BS. Evaluación del rendimiento masticatorio en pacientes portadores de sobredentaduras mandibulares retenidas por implantes oseointegrados. Trabajo de investigación Requisito para optar al título de Cirujano-Dentista. Universidad de Chile, Facultad de Odontología. Santiago, 1996.

51. Palma Saavedra IA. Rendimiento masticatorio en pacientes rehabilitados con prótesis fija dentosoportada. Trabajo de investigación Requisito para optar al título de Cirujano-Dentista. Universidad de Chile, Facultad de Odontología. Santiago, 2001.

52. Salazar Muñoz CM. Rendimiento masticatorio en pacientes rehabilitados con prótesis fija implanto soportada. Trabajo de investigación Requisito para optar al título de Cirujano-Dentista. Universidad de Chile, Facultad de Odontología. Santiago, 2001.

53. Schiattino Cueto FM. Eficiencia masticatoria en dentición natural en relación a la pérdida de unidades funcionales oclusales. Trabajo de investigación Requisito para optar al título de Cirujano-Dentista. Universidad de Chile, Facultad de Odontología. Santiago, 2001.

54. Quinteros ME. Estudio comparativo del rendimiento y la fuerza masticatoria máxima funcional en pacientes portadores de prótesis parcial removible dentomucosoportadoras y dentoimplantosoportadoras. Trabajo de investigación Requisito para optar al título de Cirujano-Dentista. Universidad de Chile, Facultad de Odontología. Santiago, 2001.

55. Cuevas Alvarado MA. Evaluación del rendimiento masticatorio e impacto en la calidad de vida en pacientes portadores de sobredentaduras mandibulares retenidas por implantes Tramonte (R) de carga inmediata. Trabajo de investigación Requisito para optar al título de Cirujano-Dentista. Universidad de Chile, Facultad de Odontología. Santiago, 2003. 